through the needle used for transfusion if the latter is connected with the apparatus by means-of an adaptor. Ideally, a few cubic centimetres of blood should be taken into a plain tube and allowed to clot, and a similar quantity should be taken into a tube containing oxalate. These samples should be kept in a refrigerator and not be discarded until the end of 24 hours after transfusion, when the patient has been seen again and been found to respond favourably to the transfusion. When the patient is seen within 24 hours after a transfusion and a haemolytic reaction is suspected, a large sterile venous sample of blood should be obtained and also a clean sample of urine. If the patient is seen later the same samples should be obtained, although many of the tests set out below will be inapplicable.

Precautions to be Observed in Obtaining the Venous Sample. -A large $(20 \mathrm{c.cm}$.) sterile syringe with a needle of not less than 20 s.w.G. should be used. The syringe and needle should be absolutely dry or else must be rinsed in sterile isotonic saline. Venepuncture must be clean. After venepuncture the needle must be removed from the syringe before the blood is squirted into the sample tubes. The sample should be partitioned between two plain tubes (one sterile) and one tube containing dry oxalate.

The Sample of Urine.-It will be sufficient to obtain a clean sample of urine, unless there is any risk of contamination with blood (in a recently delivered woman, for instance); in such cases a catheter sample must be obtained.

\section{Examination of the Samples}

In an obscure case the majority of the following tests may have to be made:

(a) Donor's Blood (from the Transfusion Bottle if Possible).(i) A sample should first be withdrawn from the bottle with a sterile pipette and kept in a sterile tube in case bacteriological examination is required. (ii) $\mathrm{A}$ mixed sample should next be centrifuged to determine the amount of free haemoglobin in the supernatant plasma. (iii) The blood should be regrouped, testing both cells and plasma; if necessary, the $R h$ group of the cells should be determined. (iv) The cells should be tested against the recipient's serum, using the pretransfusion sample of the latter when available. (v) The titre of the agglutinins in the donor's plasma should be estimated if necessary.

(b) The Recipient's Blood (Post-transfusion Sample).-(i) The sample in the sterile tube should be submitted for bacteriological examination. (ii) The serum from the second clotted sample should be examined by naked eye for the presence of haemoglobin or excess bilirubin. If there is no obvious pinkness the serum should be examined spectroscopically. The amount of bilirubin or the icterus index should be estimated, and Schumm's test for methaemalbumin should be applied. The examination of the pre-transfusion sample, when available, is a valuable control. A small amount of serum should be reserved for serological tests. (iii) The oxalated sample should be divided : one-half should be centrifuged, so that the plasma can be examined for pigments as a check on the examination of the sample of serum; the other half should be kept for serological tests. (iv) Serological Tests.-(a) A suspension of the recipient's blood should be prepared by adding one drop of blood to approximately $2 \mathrm{c.cm}$. of isotonic sodium citrate or saline (to make a $2 \%$ suspension approximately). This suspension should be examined under the low power of the microscope for the presence of agglutinates. With experience it is possible to recognize the difference between weak diffuse clumping due to auto-agglutination and the presence of small scattered well-formed agglutinates lying in a field of free cells, the latter appearance being due to the presence of clumps of donor cells in the suspension. (b) The blood should be regrouped, testing both cells for agglutinogens and serum (the latter from the clotted sample) for agglutinins. If necessary, the $R h$ group of the cells should be determined and the serum examined for the presence of anti-Rh agglutinins. $(c)$ The agglutinins in the serum should be titrated for comparison with the results of titrations carried out on samples obtained later and on the pre-transfusion sample (when available). As explained above, characteristic changes occur after the transfusion of incompatible blood, and if a sample of the donor's blood is not available, or if there is any question of the faulty labelling of a bottle so that the true donor is not known for certain, this investigation may be invaluable. (d) If necessary, differential agglutination tests should be carried out.

Note on Methods of Differential Agglutination.-Application of the technique of differential agglutination permits the recognition, in a sample of blood taken from the recipient, of foreign red cells derived from a previous transfusion. The donor cells can be recognized either by being agglutinated by a test serum which does not agglutinate the recipient's cells, or by their failure to be agglutinated by a test serum which completely agglutinates the recipient's cells For example, when the donor is Rh-positive and the recipient Rh-negative, by testing with an anti-Rh serum the donor's cells (if surviving) can be recognized as discrete clumps lying in a sea of free cells. Alternatively, when the donor belongs to group $\mathrm{O}$ and the recipient to group $\mathbf{A}$ the recipient's cells can be completely agglutinated with an anti-A serum and the free (donor) cells can then be counted in a haemocytometer. Many further possibilities of -differentiation are available if anti-M and anti- $\mathrm{N}$ test sera are also used.

Application of the method of differential agglutination is practically essential for the solution of many transfusion problems, but reliable results can only be obtained with considerable experience. For a description of methods see Wiener (1942) and Dacie and Mollison (1943):

(c) Sample of Recipient's Urine.-The urine should be examined by naked eye and its colour noted; it should then be examined spectroscopically for oxyhaemoglobin and methaemoglobin. If the supernatant fluid contains no pigments a sample should be centrifuged and the deposit examined for the presence of pigment casts. The urine should also be examined for the presence of urobilin (Schlesinger's test). The usual tests for specific gravity and for albumin should be carried out and the reaction determined.

(The concluding part, with case reports and a list of references, will appear in next week's issue)

\section{THREE CASES OF PROLONGED FAECAL FISTULA FOLLOWING OPERATION FOR APPENDICITIS}

\author{
P. T. CRYMBLE, F.R.C.S. \\ Professor of Surgery, Queen's University, Belfast
}

A slight temporary faecal fistula following the removal of a gangrenous or perforated appendix is not an uncommon occurrence, but in our experience it closes naturally in a few weeks. One may look for it in those cases in which the inflammation has spread on to the wall of the caecum, or in which closure of the appendix stump has been unsatisfactory. In the past 15 years three persistent and complete faecal fistulae following appendicectomy operation have been encountered, the duration being one, three, and four and a half years. Once the fistula had become established no motions were passed by the normal route, there was marked incapacity for work, and the social result was appalling.

The first case was that of a child of 6 who was brought to Queen Street Children's Hospital 15 years ago. The child was wasted, anaemic, in a filthy condition, and had been confined to the house for a year. Its condition did not permit of attendance at school. There was a history of two operations -one for removal of the appendix, with drainage of an abscess ; and a second one for the cure of an incisional hernia. It was after this second operation that the faecal fistula appeared.

On examination we found the ileo-caecal valve opening on the surface of the right iliac fossa and discharging squirts of fluid faeces. The entire caecum appeared to be absent, and it was decided that a local closure was impossible. After some building up, the abdomen was opened through the left rectus, and a diversion route established in the following way: terminal ileum divided, and both ends closed ; transverse colon divided, and both ends closed; lateral anastomosis between the proximal end of the ileum and the distal end of the transverse colon.

As a result of this severe operation the child had a stormy period of five days, but recovered, and was left with a mucous fistula in place of a faecal fistula. The child returned home, put on weight, recovered strength, and was able to attend school. She attended occasionally at the out-patient department, but one was inclined to postpone the removal of the isolated bowel for some years. However, our hands were forced, as some six months after her diversion operation she walked into the out-patient department with the entire ascending colon prolapsed through the fistula and hanging down under her skirt. She was admitted to hospital, the fistula circumcised, and the terminal ileum, ileo-caecal junction, and ascending 
colon removed. The operation turned out to be extremely easy, as the bowel had already mobilized itself. A few vessels were ligatured and divided and the isolated bowel was removed. There was no reaction, and the wound healed in one week.

The specimen was mounted, and is in the Queen's University museum. It shows a very atrophic terminal ileum (usually mistaken for an appendix by the students in the final examination), the fistulous opening surrounded by a ring of skin and the ascending colon (Fig. 1).

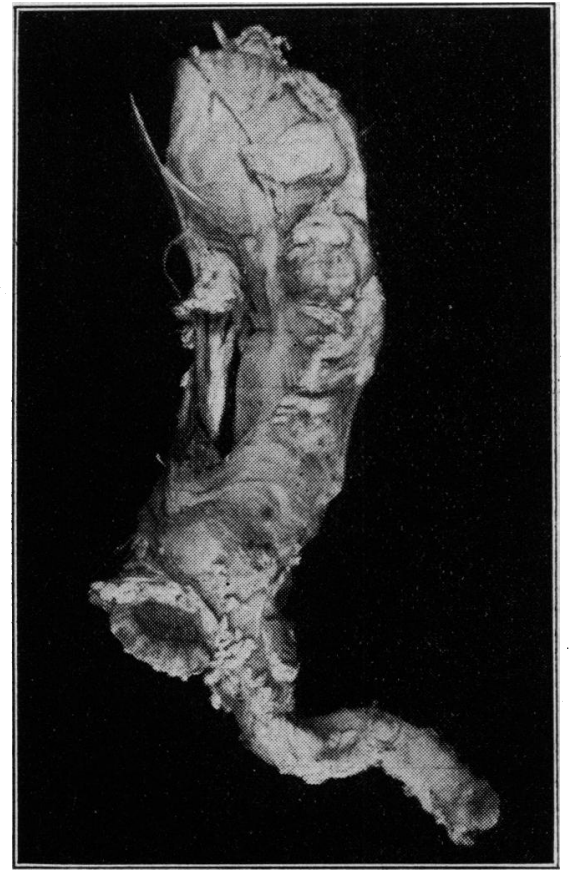

Fig. 1.-The terminal ileum, caecal fistula, and ascending colon removed from a child ased 6 years. Note the atrophic terminal ileum and the almost complete absence of the caecum.

\section{Case II}

A young man aged 19 was admitted to the Royal Hospital on Feb. 14, 1937, with a faecal fistula in the right iliac fossa which had been present for three years. A gangrenous appendix had been removed in March, 1934, and a drainage tube had been left in for nine days. The faecal fistula followed the removal of the tube, and persisted. His general condition was quite good, and he was under the impression that a faecal fistula was the usual result of the operation he had undergone. He came to the Royal because he desired to join one of the Services, and had been told that he could not be accepted in his condition. All his motions passed by the fistula, but he kep reasonably clean by packing wool into the opening. Examination of the fistula revealed that it was large bowel, and it was thought that a local closure might succeed.

Technique of Operation.-Fistula circumcised and closed by suturing the skin edges together. Clean towels, gloves, and instruments supplied. Peritoneal cavity opened and caecum separated from the anterior abdominal wall. Narrow-bladed Schoemaker clamp applied to long axis of caecum and the muco-cutaneous tissue distal to the clamp removed, leaving a cuff of bowel wall distal to the clamp. The cuff was then sutured, the clamp removed, and the suture line enfolded.

The wound healed by first intention. The patient had a natural motion on the fifth day, and left hospital on March 3, 1937, with a normal alimentary canal. It was fortunate in this case that there was enough caecum left to permit of closure without in any way obstructing the ileo-caecal valve.

\section{Case III}

This case was that of a young unmarried woman aged 19 who had passed nothing by the rectum for four and a half years. In June, 1937, after an operation for appendicitis, a faecal fistula developed, and since then no faeces had ever passed by the rectum. In 1939 three attempts were made to cure the condition, but they were unsuccessful. The first attempt was a lateral anastomosis between two loops of ileum, but this produced no change in the fistula. In the second the two bowel loops distal to the anastomosis were divided and closed, but again with no improvement. (These two operations were presumably done under the impression that the fistula was purely ileal, and involved a portion of bowel some distance from the valvula coli.) The third operation was a direct attack on the fistula, and as it caused complete intestinal obstruction it had to be reopened. She left hospital in fair condition with a complete faecal fistula.

On Jan. 14, 1941, she was admitted to our unit with a history of having four fluid mations daily. She had done no work

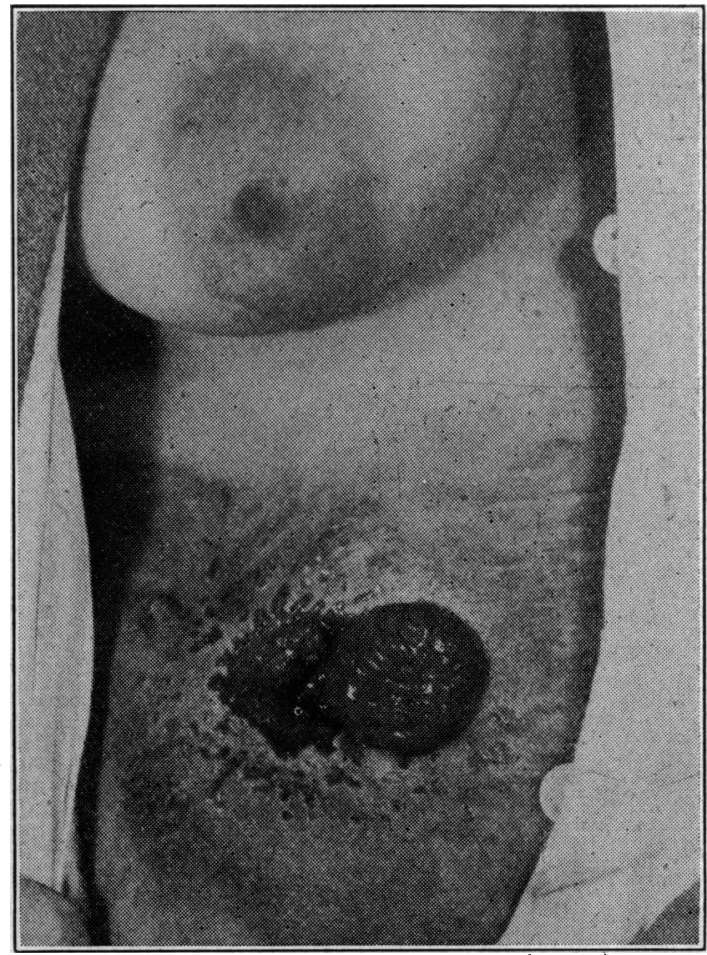

Fig. 2.-The right side of the abdomen in Case. HI. Note the extensive area of ulcerated skin surrounding the large fistula. Three weeks after the short-circuit operation this skin was entirely healed.

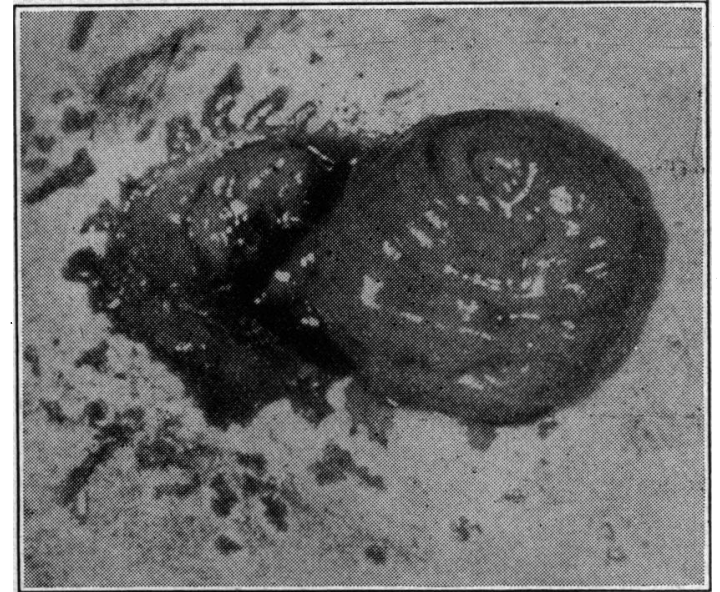

Fig. 3.-An enlarged view of the faecal fistula in Case III. The medial half of the fistula consisted of the prolapsed end of ileum. The lateral half was mainly colonic mucous membrane. Note the ulcerated skin.

but led a fairly normal life. She walked and went to the pictures. There was no emaciation, but she was pale in colour. The whole right iliac fossa showed a skin stippled with small ulcers and areas of white (Figs. 2 and 3). The actual fistula was a complicated arrangement of openings surrounded by a very tender cutaneous margin. The medial half was occupied by the prolapsing end of small bowel (ileum), which discharged no faeces and no gas. The entire index finger could be inserted into it. The lateral half showed two openings, both of which discharged faeces. The upper one could be seen, and it could 
be explored with the finger. 'Its mucous membrane was much paler than the ileal mucous membrane and had the appearance of colonic mucosa. The lower one was too tender to permit examination, and we were uncertain whether it was large or small intestine. Medial to the fistula were two incision scars, one midline and the other right rectal.

Rectal examination revealed a plug of mucus in the ampulla. The patient wore a colostomy belt, which included a large celluloid cup. She was very depressed, had no hope of cure, and showed little enthusiasm for further operative treatment.

Procedure.-The first point was to establish the nature and anatomy of the bowel distal to the fistula. One wanted to be certain that the bowel, which had not been used for four and a half years, was patent, and that the pelvic colon was not involved in the fistula. Olive oil was injected into the rectum and escaped from the two lateral fistulae. Two days later a water enema escaped from the same two fistulae. An opaque enema was then given, and this outlined the colon up to the middle of the ascending colon. It showed a bowel narrow but otherwise normal (Fig. 4). On Jan. 21 the abdomen was

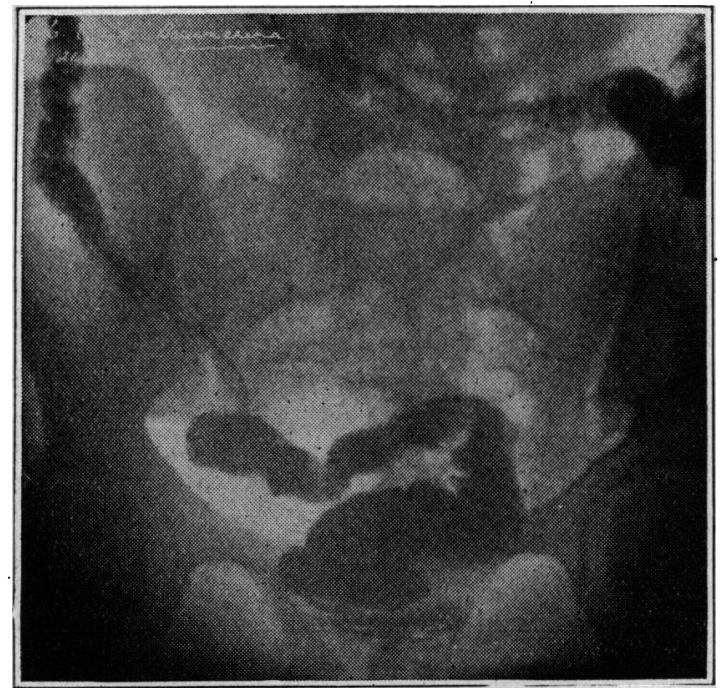

FIg. 4-Opaque enema of the third case of faecal fistula. The whole colon up to the hepatic flexure is outlined. The pelvic colon has no connexion with the fistula, and the transverse colon is very narrow. This colon had not functioned for four and a half years.

opened through a left rectus incision. No adhesions were found in this region, and there was no difficulty in tracing the small bowel from the beginning of the jejunum to that part of the lower ileum which was adherent to the region of the fistula. This portion of the ileum was divided and the two ends closed. The transverse colon was then withdrawn. It was somewhat small and atrophic, and the great omentum was reduced to a short apron about two inches long. The transverse colon was divided to the right of the middle line and the two ends closed. A side-to-side anastomosis was carried out between the proximal ileum and the distal transverse colon.

Post-operative Course.-Small quantities of fluid were given by the mouth, and there was constant vomiting for five days. On the 25 th she had $53 \mathrm{oz}$. by mouth and vomited $26 \mathrm{oz}$. On the 26 th she had $38 \mathrm{oz}$. by mouth and vomited $14 \mathrm{oz}$. The pulse remained good, and ran between 84 and 100 . There was slight evening pyrexia. Constant lower abdominal pain was felt, and the patient remained depressed and irritable.

The fistula was dressed for the first time on the 25 th, and was found to be covered with a quantity of faeces. It was thought that this had been extruded from the isolated bowel. The lower abdomen was somewhat distended. On the 27 th a little flatus was passed, the pulse and temperature had fallen, the abdominal pain was much less, and the patient for the first time was cheerful. Subsequently the left rectus incision discharged a little faeces for a few days, but it had completely closed by Feb. 5. This was an indication that the ileo-colic anastomosis had leaked. At first there was no control over the rectal discharge, but rapidly control was regained, and by Feb. 7 there was normal control of the sphincters. She left hospital in good condition on Feb. 25. The left rectus incision was healed. The skin around the fistula was free from ukeration, and the fistulous area had contracted and was discharging a little mucus.

\section{Lessons from these Three Cases}

1. All were cases of the "late appendix" which required drainage, and the condition would never have arisen if the appendicitis had been diagnosed in the first twenty-four hours.

2. If drainage is necessary, never use anything more rigid than a strip of corrugated rubber or a little piece of gauze. A rigid piece of rubber tubing left in for nine days may quite easily perforate the wall of the caecum.

3. Great care must be exercised in handling inflamed or adherent gut, as it is quite easy to tear or rupture such intestine. If the search for the appendix endangers the gut, it is better to leave the appendix in situ and content oneself with drainage.

4. It is remarkable how well a patient may be without the use of the colon. The man aged 19 was in excellent condition, and was well enough to desire to join one of the Services. He was not even depressed with his condition, and kept himself reasonably clean with a large plug of cotton-wool packed into the ascending colon. The woman (Case III) was in fairly good health, and, apart from the fact that she did no work, led a normal life. The surrounding skin in her case was a constant source of trouble.

5. Local closure should only be attempted when the fistula involves the caecum or ascending colon and is some distance away from the valvula coli. For example, the planned caecostomy is the ideal one for local closure.

6. In the presence of multiple openings or where the fistula is close to the valvula coli, one should sacrifice the whole right colon and ileo-caecal region by carrying out a two-stage operation: first a diversion operation, and later a right colectomy.

7. In Case III the essential points were the condition of the colon distal to the fistula and the condition of the small bowel proximal to the fistula. The first point was settled by the injection of olive oil into the rectum (this showed a clear route between the rectum and the fistula) and an opaque enema (which showed non-involvement of the pelvic colon). The second point had to be demonstrated at operation. Had there been numerous adhesions in the left side of the abdomen one might have found it impossible to determine the upper from the lower end of a coil of bowel. Fortunately there were no adhesions, and this problem did not arise.

\section{Classification of Faecal Fistulae}

(a) Slight temporary fistulae following appendicitis operations - These require no treatment, and tend to heal in two or three weeks.

(b) Fistulae following bowel resection.-These arise at the anastomosis or at the closed bowel end, are usually partial, and close naturally. It is easier for the faeces to pass along the mucous channel than to overcome the fibrotic contraction of the fistula.

(c) Injury to bowel during operations on adjacent viscera.Long-standing gall-bladder or kidney disease may lead to adhesions and weakening of the bowel wall. The removal of the gall-bladder or kidney may leave a weak area of bowel wall unsupported, or the bowel may be injured by the surgeon's manipulations, with the result that a few days after the operation a fistula develops. In one case in which the duodenum was involved death resulted. In a second case the ascending colon gave way after the removal of a very adherent tuberculous right kidney. This fistula closed naturally, but was succeeded by a purulent discharge of some months' duration.

(d) The operation of caecostomy. -This can be closed by a local operation when the distal route has been restored.

(e) The gross permanent fistula involving the caecal wall, some distance from the valvula coli, following an appendix operation.-This is suitable for local closure as in class $d$.

$(f)$ The gross permanent fistula in the immediate neighbourhood of the valvula coli or the fistula with several openings. This should be treated by a two-stage operation. In stage 1 a short sircuit is performed by dividing the ileum (as low down as possible), dividing the transverse colon (closing the distal end), and performing an end-to-side anastomosis between the proximal ileum and the distal transverse colon. This converts the faecal fistula into a mucous fistula. In stage 2 a right colectomy is performed. 


\section{Conclusion}

A final word of warning with regard to purulent or faecal fistulae in the region of the terminal ileum is that there is a tendency for some of these patients to develop small-bowel obstruction after even slight abdominal operations. They would appear to be balanced on the threshold of obstruction, and are pushed over the edge by any manipulations in that region. It is possible that such catastrophes would be avoided by a preliminary short circuit between the ileum and the transverse colon.

\section{COMPLACENCY IN RESUSCITATION OF THE DROWNED}

BY

\section{F. C. EVE, M.D., F.R.C.P.}

The manuscript directions for resuscitation by the Schäfer method are in the hands (copyright) of the Royal Life Saving Society, who still regard the method as completely adequate. But knowledge of the very intricate subject of respiration has naturally advanced much since Schäfer's day, and an attitude of complacency will cause us to drift behind other countries. An excellent paper by Surg. Lieut. G. H. Gibbens (1942) has given us a useful jog. He had been bitterly disappointed with Schäfer's method when he used it in his ship on drowning cases-now so urgent a problem. Instead of the normal elastic inspiration on removing pressure to which he was accustomed in normal subjects, the victim's abdomen and thorax "felt like putty." Schäfer's method relies for inspiration on elastic recoil, and this was absent owing to lack of muscular tone. He therefore adapted to ships my rocking method (Eve, 1932), in which a dozen times a minute the patient (lashed to a stretcher) is rocked, face downwards, through a total angle of 60 to 90 degrees on a pivot-provided either by a trestle or by a rope from hammock hooks passed under the middle of the stretcher. On this see-saw the weight of the abdominal contents pushes and pulls the diaphragm alternately up and down by a piston action which is actuated by gravity and does not depend on elastic recoil for inspiration. The ventilation thus secured is quite adequate, and is equal to Schäfer's in the normal subject. (Killick and Eve, 1933). The air can be felt, and heard, passing in and out freely; there is no need for voluntary breathing. A manual method is used till rocking has begun. On shore, a builder's twowheel hand-cart would do well for rocking. Prof. Cordier of Belgium points out that in Scandinavia and Eastern Europe Silvester's method-in spite of its disadvantagesis considered more efficient, especially when tested on the warm cadaver which is toneless. They advance excellent reasons for this preference, which are too lengthy to quote here.

\section{Schäfer's Method}

On reading Schäfer's (1907-8) splendid Harvey lecture I see that he started experiments in the dead-house, but found rigor mortis an insuperable difficulty; warm cadavers are not mentioned, nor are drowning cases. Then he proceeded to anaesthetized dogs (drowned) and afterwards to normal humans. Speaking of his method, he says that you should "press gradually for three seconds with both hands close together flat on the back over the loins; the fingers extending over the lower ribs. .... Of course, on relaxing the pressure the same volume of air must pass in again as the chest resumes its former shape and size."

Unfortunately, this perfectly natural assumption, justified by his experiments on normal people, does not hold good in the nearly drowned, in whom the elastic tone of the respiratory muscles fails progressively. Later, he modified his directions so as to compress the diaphragm only; but with large hands and small loins the ribs cannot always be avoided-neither, in my opinion, can it be an important point when fighting to save a life.

\section{Other Imperfections of Schäfer's Method}

The fact is that Schäfer's method has many well-known advantages and may be the best manual method; but it is not a perfect method, and it is deplorable complacencyespecially in these days of $U$ boats-not to make every effort to find a better one. We must think not so much of the very numerous successes of Schäfer's method but rather of the distressingly greater number in which it fails, and try to diminish this. A drawback of Schäfer's method is that the weight of the shoulders and upper back opposes inspiration. This, in other countries, has been met by a second operator, who lifts the folded elbows (arms extended high over the head) upwards from the ground during inspiration - thereby greatly improving ventilation and circulation (Hederer). Radiographs have recently shown (Barclay) that in the prone position the diaphragm is pushed up into the full expiratory position, and this second drawback would be increased in the toneless body. This is partly countered (Henderson, 1937) by extending the arms above the head, but both of these methods rely on muscle tone.

The main defect in the nearly drowned is muscular tone, both for circulation and for respiration. It is therefore useless to judge any resuscitation method by its effects on normal persons: the best criterion is the toneless warm cadaver, and I have rocking stretchers at various hospitals to test this aspect.

\section{Tone of the Diaphragm}

The diaphragm is the chief and only indispensable muscle of respiration. It achieves inspiration by contracting its high dome into a low dome. In the warm cadaver, as it has lost all tone, the diaphragm is pulled fully up by the elastic contraction of the lungs, and by the pressure of the abdominal contents.

In the semi-final stages of incomplete death, of the three methods of resuscitation Schäfer's will be the first to become impotent with the loss of elasticity (due to tone of the diaphragm), on which he relies for inspiration; Silvester's method will still have some elastic recoil around the ribs to rely on ; the piston action of my rocking method should work even more freely as the tone of the diaphragm declines. How swiftly this may happen was shown by H. W. Haggard (U.S.A.), who tried artificial respiration on a man who had just had a fatal heart attack. Ventilation of the lungs was normal at first, but after 10 to 15 minutes it failed completely. The asphyxiated nerve cells of the bulb and spinal cord which maintain the muscles in tone had died, so that the lungs collapsed in the toneless thorax. Henderson (1937) demonstrated another instructive point by measuring the total air respired in a quarter of an hour by various students. Then he tried artificial respiration on them and found the same figure, however hard the operator tried to increase the ventilation of the lungs. The respiratory centre-amazingly sensitive to $\mathrm{CO}_{2}$ - knew exactly how much ventilation was necessary, and by modifying the tone of the diaphragm set the stroke of its piston to give the correct ventilation both in natural and in artificial respiration.

\section{Attempting Better Methods}

The trouble with apparatus for the rocking method is that it may not be at hand when needed; but, in a ship, a stretcher which can be rocked could be instantly available, and if resuscitation drill is practised the interval between the victim's lying on the deck and rocking on the stretcher will already have been reduced-with my later gadgets-to 25 seconds. I find, from the officer responsible for first aid in one of the small ships (a mine-sweeper) which carry out many of the rescues from drowning in convoy, that there is no difficulty-as he showed me-in storing, suspending, and rocking a stretcher; he was enthusiastic about it. The short boat journey to the ship is the weak but vital stage. Thwarts and crowding may render the Schäfer method impossible in a boat. So our St. John men at Beverley are trying rocking each other on the four bent forearms of two men (opposite), with their two pairs of legs steadied by a thwart between them. Improvements 